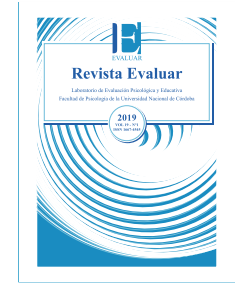

\title{
Validación de la Escala de Actitud hacia la Investigación en Estudiantes Mexicanos de Psicología
}

\author{
Validation Study of the Attitude Scale towards Research among \\ Mexican Psychology Students
}

\author{
Lucía Quezada-Berumen * ${ }^{1}$, José Moral de la Rubia ${ }^{1}$, René Landero-Hernández ${ }^{1}$ \\ 1 - Facultad de Psicología, Universidad Autónoma de Nuevo León, Monterrey, NL., México.
}

\author{
Introducción \\ Método \\ Resultados \\ Discusión \\ Referencias
}

Recibido: 02/02/2019 Revisado: 21/02/2019 Aceptado: 25/02/2019

\begin{abstract}
Resumen
Esta investigación tiene como objetivo validar la Escala de Actitud hacia la Investigación. Se usó un muestreo de bola de nieve. Participaron 392 estudiantes de psicología de una universidad pública del noreste de México, 74.2\% mujeres y $25.8 \%$ hombres. El análisis factorial exploratorio arrojó una estructura de tres factores: afectivo-conductual de ítems directos o redactados en sentido de aceptación $(\alpha$ ordinal $=.90)$, cognoscitivo $(\alpha$ ordinal $=.89)$ y conductual-afectivo de ítems inversos o redactados en sentido de rechazo ( $\alpha$ ordinal $=.87$ ). El ajuste del modelo fue bueno $(\mathrm{NFI}=.95, \mathrm{RFI}=.95, \mathrm{RMSEA}=.02 \mathrm{y} \mathrm{SRMR}=.07)$ y los tres factores mostraron validez convergente (AVE $>.50$ y $\omega$ $>.80)$ y discriminante $\left(r_{\mathrm{F} 1 \mathrm{~F} 2}^{2}<\mathrm{AVE}_{\mathrm{F} 1}\right.$ y $\left.\mathrm{AVE}_{\mathrm{F} 2}\right)$. La actitud promedio fue favorable hacia la investigación sin diferencia entre mujeres y hombres, salvo por un mayor promedio en el factor afectivo-conductual de ítems directos en hombres. El puntaje promedio más alto en estudiantes que piensan dedicarse a la investigación y la correlación positiva entre la escala y la calificación en la materia de investigación proporcionaron evidencias de validez de constructo. Se concluye que la escala presenta consistencia interna y validez de constructo.
\end{abstract}

Palabras clave: actitud, investigación, análisis factorial, confiabilidad, México

\begin{abstract}
The aim of this research was to validate the Scale of Attitude towards Research (SAR). A snowball sampling was used. A sample of 392 psychology students, 74.2\% women and $25.8 \%$ men, from a public university in northeastern Mexico was collected. The exploratory factor analysis yielded a structure of three factors: affective-behavioral factor of positively codified items, phrased in the sense of acceptance (ordinal $\alpha=.90$ ), cognitive factor (ordinal $\alpha=$ .89), and behavioral-affective factor of negatively codified items, phrased in the sense of rejection (ordinal $\alpha=.87$ ). The model had a good fit (NFI $=.95, \mathrm{RFI}=.95$, RMSEA $=.02$, and SRMR $=.07)$ and the three factors showed convergent validity (AVE $>.50$ and $\omega>.80)$ and discriminant validity $\left(r_{\mathrm{F} 1, \mathrm{~F} 2}^{2}<\mathrm{AVE}_{\mathrm{F} 1}\right.$ y $\left.\mathrm{AVE}_{\mathrm{F} 2}\right)$. The average attitude was favorable towards research, with no difference between women and men, except for a greater average in the affective-behavioral factor of positive-codified items among men. The highest average on the scale among students who plan to pursue the research area and the positive correlation between the scale and the marks in a research related subject provided evidence of construct validity. We concluded that the scale shows internal consistency and construct validity.
\end{abstract}

Keywords: attitude, research, factor analysis, reliability, Mexico

Cómo citar este artículo: Quezada-Berumen, L., Moral de la Rubia, J., \& Landero-Hernández, R. (2019). Validación de la Escala de Actitud hacia la Investigación en estudiantes mexicanos de psicología. Revista Evaluar, 19(1). Recuperado de https://revistas.unc.edu.ar/index.php/revaluar

*Correspondencia a: Lucía Quezada-Berumen. Universidad Autónoma de Nuevo León (UANL), Facultad de Psicología. Dr. Carlos Canseco 110. Col. Mitras Centro. C. P. 64460. Monterrey, Nuevo León, México. Tel. 005281 83338233. Ext. 208. E-mail: luciaqb86@msn.com 


\section{Introducción}

La investigación es una actividad orientada a la búsqueda de nuevos conocimientos en cualquier campo del saber humano. Tradicionalmente, la investigación ha sido una actividad atribuida principalmente a las instituciones de educación superior, con el propósito de responder a las necesidades de la sociedad (De la Cruz, 2013; González-González, Galindo-Miranda, Galindo-Miranda, \& Gold-Morgan, 2004). Si bien es cierto que el objetivo fundamental de la educación en pregrado no tiene como función específica formar investigadores, a este nivel educativo sí le corresponde fomentar en sus alumnos actitudes positivas hacia la investigación, de manera que se conviertan en sus usuarios y, en el mejor de los casos, la adopten como una forma habitual de afrontar los problemas que les plantea el ejercicio de su profesión (Aldana, Caraballo, \& Babativa, 2016; Ruiz, 2010).

Una de las razones por las que la investigación es importante en los centros docentes es porque estimula el pensamiento crítico y la creatividad. Así, esta constituye un medio por cual el proceso de aprendizaje se vitaliza y permite combatir el aprendizaje por memorización, el cual tanto ha contribuido a formar profesionales pasivos, sin innovación y con escasa curiosidad e iniciativa personal (Ruiz, 2010). La falta de interés y las actitudes negativas de los estudiantes hacia la ciencia y la tecnología plantean un problema para la educación científica, ya que llevan a conocimientos deficientes sobre la ciencia y una falta de iniciativa e implicación en proyectos científicos, los cuales son necesarios para que el sistema de ciencia y tecnología mantenga su actividad de progreso (Vázquez \& Manassero, 2008).

Las actitudes hacia la investigación se pueden considerar tanto causa como efecto, esto es, como determinantes y objetivos del aprendizaje en materias de investigación. Como causas del aprendizaje, las actitudes positivas lo favorecen y las negativas lo dificultan; como efecto del aprendizaje, las actitudes pueden ser un contenido más de aprendizaje $\mathrm{y}$, por tanto, ser enseñadas y aprendidas en la universidad e instituciones de educación media superior. Una buena actitud hacia el estudio y el aprendizaje en general suele ser garantía de motivación, interés y esfuerzo en los estudiantes. A su vez, los profesores entienden y aprecian las actitudes en este sentido como elementos que favorecen el rendimiento en las materias de investigación. Sin embargo, las actitudes también pueden ser planteadas como una consecuencia o efecto de la educación, es decir, como objetivos a alcanzar por medio de esta (Vázquez-Alonso \& Manassero-Mas, 1997).

Hasta el momento se ha hablado de la actitud hacia la investigación científica, pero falta definir este constructo en el cual se centra el presente estudio instrumental. La actitud hacia la investigación científica es una organización duradera y persistente de creencias, sentimientos y disposiciones respecto a la investigación por parte de los individuos de un colectivo (Aldana de Becerra \& Joya-Ramírez, 2011). De acuerdo con Estrada-Corona (2012), si se conocen las actitudes manifiestas de una persona hacia el objeto de actitud es posible inferir cuál será su conducta.

Existen algunos instrumentos para evaluar las actitudes hacia la investigación, como la Escala de Actitudes hacia la Investigación desarrollada por Papanastasiou (2005) en Chipre. En Venezuela, se encuentra la Escala de Actitud hacia el Proceso de Investigación Científico-Social en el docente investigador universitario de Blanco y Alvarado (2005). En Colombia, se han desarrollado la Escala de Actitudes hacia la Investigación Científica en Docentes de Metodología de la Investigación por Aldana de Becerra y Joya-Ramírez (2011); y más recientemente la Escala para 
medir Actitudes hacia la Investigación (EACIN) en ambientes académicos creada por Aldana et al. (2016).

Con respecto a esta última escala, su validez de contenido ha sido establecida a través de un juicio de expertos y se ha demostrado su confiabilidad a través de la consistencia interna (Aldana et al., 2016). La escala fue diseñada para medir tres aspectos o factores interrelacionados de la actitud: afectivo, cognoscitivo y conductual. El factor afectivo refiere a lo que la persona siente y a las emociones que le produce la investigación; el cognoscitivo, a lo que la persona sabe o cree saber acerca de la investigación; y el conductual, a lo que la persona hace o está dispuesta a hacer con respecto a la investigación (Aldana de Becerra \& Joya-Ramírez, 2011). Cabe señalar que este modelo de tres factores correlacionados no ha sido demostrado empíricamente.

Por su versatilidad para evaluar las actitudes hacia la investigación en comunidades académicas, su desarrollo en Latinoamérica y su contenido, la EACIN se considera un instrumento adecuado para medir las actitudes hacia la investigación en estudiantes universitarios mexicanos. Contar con este instrumento facilitaría obtener un mayor conocimiento y un mejor abordaje de la implicación de los estudiantes en actividades científicas dentro de las Instituciones de Educación Superior, las cuales están haciendo un énfasis creciente en la importancia e incorporación de la investigación científica dentro de sus programas educativos. No obstante, sus propiedades métricas no han sido estudiadas en México y el establecimiento de su validez de constructo está en proceso.

Por lo antes mencionado, el propósito de este estudio fue validar la EACIN y medir la actitud hacia la investigación en estudiantes de psicología de una universidad pública mexicana. Se plantearon seis objetivos: 1) valorar las propiedades de distribución, discriminabilidad y consistencia interna de los ítems, 2) explorar la estructura factorial verificando que sus factores presenten consistencia interna, validez convergente y validez discriminante; 3) describir la distribución de la puntuación total de la EACIN y sus factores; 4) comparar las medias entre los factores e interpretar el nivel actitudinal; 5) evaluar la relación entre la puntuación total de la EACIN y sus factores con la edad, el sexo y el semestre que se cursa y 6) probar la validez de constructo verificando la correlación positiva de la escala con la calificación en la materia de investigación, y el mayor promedio del puntaje en la escala de los estudiantes que se piensa dedicar a la investigación, en comparación con los que se piensan dedicar a otras áreas.

\section{Método}

El presente estudio empírico de tipo instrumental se realizó utilizando un diseño ex post facto de corte transversal, ya que no se manipuló ninguna variable y los datos se colectaron en un único punto temporal (Ato, López, \& Benavente, 2013).

\section{Participantes}

La población de la que se extrajo la muestra fue compuesta por estudiantes de psicología de una universidad pública del estado de Nuevo León, México. Los criterios de inclusión fueron: estar matriculado en el programa de licenciatura en psicología, cursar o haber cursado al menos una materia de investigación (hay una materia en cada semestre) y otorgar el consentimiento informado. Los criterios de exclusión fueron estudiar 
la licenciatura en otra facultad $\mathrm{u}$ otra universidad y ser estudiante de posgrado. El criterio de eliminación fue tener datos incompletos en la EACIN. Se usó un muestreo no probabilístico guiado por el participante o de bola de nieve y se obtuvo una muestra válida de 392 participantes, lo que representó el $10.5 \%$ de la matrícula total de la licenciatura en psicología de la universidad en la que se hizo el estudio ( $\mathrm{N}=3,749$ estudiantes).

De los 392 participantes, 291 (74.2\%) fueron mujeres y 101 (25.8\%) hombres. La edad osciló entre 17 y 46 años con una media de 19.41 (DE = 2.93). La media de la calificación en la materia de investigación fue 88.24 ( $\mathrm{DE}=8.07)$, variando de 40 a 100 . El $42.4 \%$ de los alumnos cursaban el primer semestre; $9.9 \%$ el segundo; $7.1 \%$ el tercero; $24.2 \%$ el cuarto; $7.4 \%$ el quinto y el 9\% correspondió a semestres del sexto al décimo. Entre las áreas de trabajo a las que el estudiante piensa dedicarse, dominaron la psicología clínica (54.8\%), educativa (17.8\%) y laboral (14.8\%). Al área de investigación pensaban dedicarse el $2.8 \%$ de los participantes.

\section{Instrumentos de medida}

Se aplicó un cuestionario en línea integrado por la solicitud de consentimiento informado, dos preguntas sobre información sociodemográfica (sexo y edad), cinco preguntas sobre información académica y la EACIN.

\section{Escala de Actitudes hacia la Investigación} (EACIN; Aldana et al., 2016). Consta de 34 ítems tipo Likert con una escala de respuesta de cinco opciones: $0=$ Muy en desacuerdo, $1=E n$ desacuerdo, 2 = Ni de acuerdo ni en desacuerdo, 3 = De acuerdo y $4=$ Muy de acuerdo. Su puntaje se obtiene sumando sus 34 ítems, tras invertir la puntuación en los ítems 1, 4, 5, 9, 14, 19, 23, $27,28,30$ y 34 , por lo que su rango varía de 0 a 136. Aldana et al. (2016) diseñaron los ítems para medir tres factores correlacionados: afectivo con nueve indicadores (ítems 2, 3, 6, 11, 14, 17, 19, 25 y 27), cognoscitivo con 12 indicadores (ítems $1,7,12,15,20,22,26,28,29,31,32$ у 33) y conductual con 13 indicadores (ítems 4, 5, 8, 9, $10,13,16,18,21,23,24,30$ y 34$)$. Su consistencia interna total es alta $(\alpha$ de Cronbach $=.85)$ y las comunalidades de sus ítems varían de .25 a .61 con una media de .45; no obstante, cinco ítems presentan propiedades de consistencia interna débiles (ítems 1, 6, 10, 18 y 31), al disminuir el valor de la $\alpha$ total con su eliminación (Aldana et al., 2016).

En el presente estudio, se añadió un nuevo ítem inverso, que se ubicó al final de la escala, sobre un aspecto conductual (ítem 35, Para ser sincero/a realmente lo que menos hago es leer). Se decidió incluirlo por dos motivos: es un aspecto señalado por los maestros de investigación en las reuniones del área y retoma la observación de que uno de los obstáculos para el aprendizaje de las materias de investigación es que los alumnos no leen (Gómez-Delgado, Bazán-Ramírez, \& Vi1lalobos-Galvis, 2017). Los puntajes de la escala y los factores se calcularon sumando los ítems puntuados en dirección hacia actitud positiva, y dividiendo por el número de ítems sumados, con lo que se obtiene un rango continuo de 0 a 4 .

\section{Procedimiento}

En los salones de clase, maestros del área de investigación invitaron a sus alumnos a que respondieran un cuestionario en línea sobre actitud hacia la investigación. Además, se solicitó a los estudiantes que, una vez terminaran de responder el cuestionario, difundieran el enlace con compa- 
ñeros a través de sus redes sociales. El enlace estuvo disponible de octubre a diciembre del 2017. Entre las instrucciones se pidió a los participantes que respondieran la totalidad del cuestionario. La participación en el estudio fue voluntaria (se solicitó un consentimiento informado para participar), no remunerada y anónima. No se registraron datos de identificación personal. Se incluyó un correo electrónico de los responsables de la investigación para resolver cualquier duda o problema suscitado por la misma, por lo que el estudio atendió a las normas de investigación de la American Psychological Association (2017).

\section{Análisis de datos}

La discriminabilidad se determinó por la diferencia de tendencia central entre el grupo de puntuaciones altas y bajas en la escala (percentil 25 y 75). El contraste se hizo por la prueba $U$ de Mann-Whitney. Se requirió una diferencia significativa y con valor mayor que .5 (un octavo del rango). Para la consistencia interna se calculó la correlación entre el ítem y el resto de la escala por el coeficiente de correlación poliserial, la comunalidad en la extracción de un factor general por el método de mínimos cuadrados no ponderados desde la matriz de correlación policórica, y el coeficiente alfa ordinal de la escala tras eliminar el ítem. Una correlación menor que .30, una comunalidad menor que $.16 \mathrm{y}$ el incremento del valor coeficiente alfa ordinal se interpretaron como indicadores de consistencia interna débil.

El número de factores se determinó por la convergencia del análisis paralelo de Horn (percentil 95 como punto de intersección), coordenadas óptimas (misma especificación) y media mínima de las correlaciones parciales al cuadrado. La extracción de factores se hizo desde la matriz de correlación policórica por el método de míni- mos cuadrados no ponderados, y la rotación de la matriz factorial por el método promax. Se requirió una carga factorial mínima de .40 en la matriz de configuraciones. Desde la matriz estructural, se calculó la varianza media extraída (AVE por sus siglas en inglés) y el coeficiente omega de McDonald o de confiabilidad compuesta $(\omega)$. Una AVE $>.50$ y un coeficiente $\omega \geq .70$ se interpretaron como indicadores de validez convergente (Borsboom, Mellenbergh, \& van Heerden, 2004). Con una AVE > .40 se puede hablar de una validez convergente adecuada, si se compensa con una mayor exigencia en la confiabilidad compuesta, como un valor de $\omega>.80$ (Borsboom et al., 2004). A su vez, una varianza compartida entre dos factores (cuadrado del coeficiente producto-momento de Pearson) menor que .66 y menor que la AVE de cada factor refleja validez discriminante entre estos (Borsboom et al., 2004). Los valores de consistencia interna de la escala y los factores se calcularon por el coeficiente alfa ordinal; valores $<.50$ se interpretaron como consistencia interna inaceptable, entre .50 y .59 pobre, de .60 a .69 cuestionable, de .70 a .79 aceptable, de .80 a .89 buena $y \geq .90$ excelente (Gadermann, Guhn, \& Zumbo, 2012).

Se estimaron los parámetros del modelo por mínimos cuadrados no ponderados, usando como datos de entrada la matriz de correlación policórica. Los intervalos de confianza al 95\% para los estimadores se calcularon por el método percentil con la extracción de 2000 muestras aleatorias. Se evaluó el ajuste del modelo a los datos a través de ocho índices: chi-cuadrada relativa $\left(\chi^{2} / g l\right)$, índice de bondad de ajuste de Jöreskog y Sörbom (GFI) y su fórmula corregida (AGFI), índice de ajuste comparativo de Bentler (CFI), índice de ajuste normado de Bentler y Bonett (NFI), índice de ajuste relativo de Bollen (RFI), error cuadrático medio de Steiger y Lindt (RMSEA), y error cuadrático medio (SRMR). Se estipularon como 
valores de buen ajuste: $\chi^{2} / g l \leq 2$, GFI, CFI, NFI y $\mathrm{RFI} \geq .95, \mathrm{AGFI} \geq .90, \mathrm{RMSEA}$ y $\mathrm{SRMR} \leq .05 \mathrm{y}$ como valores aceptables: $\chi^{2} / g l \leq 3$, GFI, CFI, NFI y $\mathrm{RFI} \geq .90, \mathrm{AGFI} \geq .85, \mathrm{RMSEA}$ y $\mathrm{SRMR} \leq .08$ (Byrne, 2016).

La normalidad se contrastó por la prueba ómnibus de D’Agostino-Pearson (Poitras, 2006). La comparación de medias entre los factores se hizo por la prueba $t$ de Student para muestras emparejadas. El tamaño del efecto se estimó por la $d$ de Cohen. Valores de $d$ menores que .2 se interpretaron como un tamaño del efecto trivial, entre .2 y .49 pequeño, entre .5 y .79 mediano y mayor que 8 grande (Téllez, García, \& Corral-Verdugo, 2015).

Las relaciones de la puntuación total de la EACIN y sus factores con la edad y calificación en la materia de investigación se calcularon por la correlación producto-momento de Pearson y con el semestre con el coeficiente por rangos de Spearman. La relación con el sexo se estableció con una comparación de medias a través de la prueba $t$ de Student para muestras independientes. El tamaño del efecto del sexo sobre la actitud hacia la investigación se calculó por la $g$ de Hedges con la corrección de sesgo, y este se interpretó como la $d$ de Cohen (Téllez et al., 2015).

La relación con el área profesional se estableció con una comparación de medias a través del análisis de varianza. El tamaño del efecto del área profesional sobre la actitud hacia la investigación se calculó por el coeficiente eta al cuadrado. Valores entre .01 y .059 se interpretaron como un tamaño del efecto pequeño, entre .06 y .139 mediano $\mathrm{y} \geq .14$ grande (Téllez et al., 2015). Se comparó al grupo del área profesional de investigación con los otros cuatro grupos de áreas profesionales a través de la prueba de Dunnett con un nivel de significación de .05. El tamaño del efecto en estas cuatro comparaciones se determinó con $g$ de Hedges con la corrección de sesgo.

Las correlaciones con la edad y el semestre cursado, ambas variables con leptocurtosis muy fuerte, se calcularon por el coeficiente de correlación por rangos de Spearman $\left(r_{\mathrm{S}}\right)$. A través de la correlación por rangos parcial $\left(r_{\mathrm{p}}\right)$ se calculó la correlación entre la escala y la edad, parcializando en ambas variables la varianza del semestre cursado, es decir, sustrayendo la covarianza que el semestre cursado tiene con ambas variables (Conover, 1999). Las correlaciones con la calificación en la materia de investigación se calcularon por el coeficiente de correlación producto-momento de Pearson y su intervalo de confianza se determinó por el método de muestreo repetitivo con la extracción de 2,000 muestras aleatorias (Hesterberg, Moore, Monaghan, Clipson, \& Epstein, 2014). Valores absolutos de $r$ o $r_{\mathrm{s}}$ entre $.01 \mathrm{y} .029$ se interpretaron como una fuerza de la asociación débil, entre .30 y .499 moderada, entre .50 y .699 fuerte y mayor o igual que .70 muy fuerte (Téllez et al., 2015).

Los cálculos estadísticos se realizaron en SPSS versión 24 (IBM, 2016) con el módulo R 4.1, Excel 2007 y AMOS versión 16. Se usó un nivel de significación de .05 con un contraste a dos colas, salvo en la prueba de normalidad, en la que el contraste fue a una cola.

\section{Resultados}

Distribución, discriminabilidad y consistencia interna de los items

Ningún ítem presentó efecto suelo o techo. El porcentaje más alto en las categorías primera y última de respuestas fue de 49.5\% ( $<80 \%$ ). No obstante, los ítems 12 y 34 mostraron asimetría positiva extrema, coeficiente de asimetría percentílico $(\mathrm{CAP})=1$ y el ítem 29 asimetría negativa 
extrema, CAP = -1; a su vez, los ítems 12, 16 y 29 presentaron una leptocurtosis (colas adelgazadas) extrema, curtosis percentílica corregida (CPC) $=.24$. El ítem 26 tuvo una variabilidad mínima, rango semi-intercuartílico $(\mathrm{RSI})=0$, y un aplanamiento (colas engrosadas) extremo, $\mathrm{CPC}=-.26$. Cabe señalar que ninguno de estos cinco ítems presentó problemas de discriminabilidad o consistencia interna, por lo que no fueron eliminados (Tabla 1).

Los ítems 28 y 32, correspondientes al factor cognoscitivo, tuvieron una diferencia media menor que .05 entre los grupos de puntuaciones altas y bajas en la escala, y mostraron una consistencia interna débil, ya que su correlación con el resto de la escala fue menor que .30, su comunalidad menor que .16 y su eliminación incrementó el coeficiente alfa ordinal de la escala, $\alpha$ ordinal $>.94$ (Tabla 1), por lo que ambos ítems no fueron incluidos en los subsiguientes análisis. La consistencia interna de la escala calculada por el coeficiente alfa de Cronbach también fue muy alta ( $\alpha$ $=.92$ ).

\section{Exploración de la estructura factorial}

El análisis paralelo de Horn, coordenadas óptimas y la media mínima de las correlaciones parciales al cuadrado y a la cuarta potencia convergieron en que el número de factores sustantivos es cuatro. Tras extraerse cuatro factores se explicó el $48.7 \%$ de la varianza de los ítems. El primer factor quedó conformado por doce ítems (ítems 2, 3, 6, 8, 10, 11, 13, 16, 17, 21, 24 y 25), el segundo por nueve indicadores (ítems $7,12,15$, 20, 22, 26, 29, 31 y 33), el tercero por dos (ítems 18 y 30) y el cuarto por diez (ítems $1,4,5,9,14$, $19,23,27,34$ y 35). Cabe mencionar que el tercer factor tuvo un número insuficiente de indicadores (ítems 18 y 30) y no mostró un verdadero contenido teórico (pues sus dos ítems son repetitivos en contenido). Por lo anterior y al mostrar menor consistencia interna en la escala, se optó por eliminar el ítem 30 (Tabla 1).

Al extraer tres factores con los restantes 32 ítems, se explicó el $45.3 \%$ de la varianza. Tras la rotación, se definió un primer factor con trece indicadores (ítems 2, 3, 6, 8, 10, 11, 13, 16, 17, 18, 21, 24 y 25), que corresponden a ítems directos de aspectos afectivos y conductuales (ACID). Su consistencia interna fue excelente $(\alpha$ ordinal $=$ .90) y una validez convergente adecuada (AVE = $.42 ; \omega=.90)$. El segundo factor quedó formado por nueve indicadores (ítems 7, 12, 15, 20, 22, 26, 29, 31 y 33) que corresponden a ítems del factor cognoscitivo (COG). Su consistencia interna fue buena ( $\alpha$ ordinal $=.89$ ) y con adecuada validez convergente $(\mathrm{AVE}=.49 ; \omega=.90) . \mathrm{El}$ tercer factor quedó integrado por diez indicadores (ítems 1, 4, $5,9,14,19,23,27,34$ y 35), que corresponden a ítems inversos sobre aspectos conductuales $\mathrm{y}$ afectivos (CAII). Su consistencia interna fue buena $(\alpha$ ordinal $=.87)$, su varianza media extraída fue próxima a $.40(\mathrm{AVE}=.39)$ y su confiabilidad compuesta fue mayor que $.80(\omega=.86)$. La varianza compartida entre pares de factores varió de .29 a .38 con una media de .32 . En todos los casos, esta varianza compartida entre pares de factores fue menor que la varianza media extraída de cada factor, por lo que los tres factores presentaron validez discriminante.

Para complementar el análisis exploratorio, se especificó un modelo de tres factores correlacionados con sus residuos independientes (Figura 1) usando la muestra de 392 estudiantes en que la se aplicó el análisis factorial exploratorio. Todas las estimaciones por intervalo fueron significativas. Los tres factores presentaron validez convergente $(\mathrm{AVE}=.64 \mathrm{y} \omega=.90$ para el factor $\mathrm{ACID}, \mathrm{AVE}=.69$ y $\omega=.89$ para el factor $\mathrm{COG}$ y $\mathrm{AVE}=.63$ y $\omega=.87$ para el factor CAII). La varianza compartida entre los factores varió de .38 a $.52(<.66)$ con una media de $.43(<.50)$, 
Tabla 1

Distribución, discriminabilidad y consistencia interna de los 35 ítems de la EACIN.

\begin{tabular}{|c|c|c|c|c|c|c|c|c|c|c|c|}
\hline \multirow{2}{*}{ Ítem } & \multicolumn{2}{|c|}{ Porcentaje } & \multicolumn{4}{|c|}{ Estadísticos descriptivos } & \multicolumn{2}{|c|}{ Discriminabilidad } & \multicolumn{3}{|c|}{ Consistencia } \\
\hline & MD & MA & Mdn & $R S I$ & $C A P$ & $C P C$ & $D M$ & $Z_{U}$ & $r_{P S}$ & $\lambda^{2}$ & $\alpha \operatorname{ord}_{\mathrm{t}-\mathrm{i}}$ \\
\hline 1 & 49.5 & 1.5 & 1 & 0.5 & 0 & -.013 & -1.239 & $-9.941^{* * *}$ & -.559 & .353 & .934 \\
\hline 2 & 3.1 & 7.9 & 2 & 0.5 & 0 & -.013 & 0.811 & $-6.369^{* * *}$ & .445 & .201 & .935 \\
\hline 3 & 5.4 & 7.4 & 2 & 0.5 & 0 & -.013 & 1.318 & $-8.385^{* * *}$ & .522 & .325 & .935 \\
\hline 4 & 21.9 & 0.3 & 1 & 0.5 & 0 & -.013 & -1.043 & $-7.796^{* * *}$ & -.474 & .251 & .935 \\
\hline 5 & 49 & 0.5 & 1 & 0.5 & 0 & -.013 & -1.195 & & -.657 & .463 & .933 \\
\hline 6 & 5.1 & 11.7 & 2 & 0.5 & .3 & -.096 & 1.589 & $-9.851^{* * *}$ & .632 & .435 & .934 \\
\hline 7 & 0.3 & 37.8 & 3 & 0.5 & 0 & -.013 & 1.115 & $-9.277^{* * *}$ & .568 & .377 & .934 \\
\hline 8 & 0.3 & 28.3 & 3 & 0.5 & 0 & -.013 & 1.152 & $-9.070^{* * *}$ & .548 & .335 & .934 \\
\hline 9 & 7.1 & 2.8 & 2 & 0.5 & 0 & -.013 & -1.049 & $-7.316^{* * *}$ & -.476 & .233 & .935 \\
\hline 10 & 1.5 & 15.8 & 3 & 0.5 & 0 & -.013 & 1.286 & $-9.564^{* * *}$ & .603 & .386 & .934 \\
\hline 11 & 1.3 & 11 & 2 & 0.5 & 0 & -.096 & 1.378 & $-9.821^{* * *}$ & .654 & .457 & .933 \\
\hline 12 & 0.3 & 47.2 & 3 & 0.5 & 1 & .237 & 0.952 & $-9.418^{* * *}$ & .534 & .324 & .934 \\
\hline 13 & 1.8 & 13.8 & 2 & 0.5 & .3 & -.096 & 1.180 & $-7.734^{* * *}$ & .509 & .253 & .935 \\
\hline 14 & 8.2 & 1.5 & 2 & 0.5 & 0 & -.013 & -1.128 & $-8.436^{* * *}$ & -.540 & .317 & .934 \\
\hline 15 & 0.8 & 47.2 & 3 & 0.5 & .2 & .031 & 0.806 & $-8.094^{* * *}$ & .443 & .244 & .935 \\
\hline 16 & 4.6 & 7.4 & 2 & 1 & 0 & .237 & 1.473 & $-8.915^{* * *}$ & .614 & .430 & .934 \\
\hline 17 & 5.1 & 9.4 & 2 & 0.5 & 0 & -.013 & 1.771 & & .741 & .607 & .932 \\
\hline 18 & 1.8 & 10.7 & 2 & 0.5 & .3 & -.096 & 1.116 & $-7.888^{* * *}$ & .506 & .268 & .935 \\
\hline 19 & 15.3 & 2 & 1 & 0.5 & 0 & -.013 & -1.415 & $-9.743^{* * *}$ & -.625 & .444 & .933 \\
\hline 20 & 0.5 & 30.6 & 3 & 0.5 & 0 & -.013 & 1.013 & $-8.670^{* * *}$ & .529 & .322 & .934 \\
\hline 21 & 0.8 & 10.2 & 3 & 0.5 & -.3 & -.096 & 1.117 & $-8.465^{* * *}$ & .506 & .287 & .935 \\
\hline 22 & 0.3 & 32.1 & 3 & 0.5 & 0 & -.013 & 1.093 & $-9.882^{* * *}$ & .614 & .422 & .933 \\
\hline 23 & 17.1 & 2.3 & 1 & 0.5 & .3 & -.096 & -1.185 & $-7.844^{* * *}$ & -.423 & .167 & .936 \\
\hline 24 & 7.7 & 4.6 & 2 & 0.5 & 0 & -.013 & 1.086 & $-6.935^{* * *}$ & .479 & .228 & .935 \\
\hline 25 & 2.8 & 5.9 & 2 & 0.5 & 0 & -.013 & 1.220 & $-8.310^{* * *}$ & .572 & .349 & .934 \\
\hline 26 & 0.3 & 21.9 & 3 & 0 & 0 & -.263 & 1.132 & -9.204 & .586 & .389 & .934 \\
\hline 27 & 12.8 & 1.8 & 1 & 0.5 & 0 & -.096 & -1.459 & $-9.745^{* * *}$ & -.634 & .426 & .934 \\
\hline 28 & 2.8 & 21.9 & 3 & 0.5 & -.3 & -.096 & 0.209 & $-2.370^{*}$ & .192 & .047 & .937 \\
\hline 29 & 0.5 & 58.7 & 4 & 0.5 & -1 & .237 & 0.845 & $-8.619^{* * *}$ & .524 & .324 & .934 \\
\hline 30 & 13 & 2 & 1 & 0.5 & .3 & -.096 & -1.165 & $-8.246^{* * *}$ & -.444 & .188 & .935 \\
\hline 31 & 0.5 & 37 & 3 & 0.5 & 0 & -.013 & 1.022 & $-9.018^{* * *}$ & .494 & .284 & .934 \\
\hline 32 & 3.3 & 13.5 & 2 & 0.5 & .3 & -.096 & 0.492 & $-3.293^{* * *}$ & .196 & .043 & .938 \\
\hline 33 & 1 & 26.5 & 3 & 0.5 & 0 & -.013 & 0.890 & $-7.472^{* * *}$ & .445 & .216 & .935 \\
\hline 34 & 8.9 & 2.8 & 1 & 0.5 & 1 & -.013 & -1.073 & $-7.854^{* * *}$ & -.477 & .234 & .935 \\
\hline 35 & 30.4 & 2.6 & 1 & 1 & .3 & .070 & -1.327 & $-8.297^{* * *}$ & -.407 & .173 & .936 \\
\hline
\end{tabular}

Nota. Porcentaje de casos para la primera opción de respuesta $(\mathrm{MD}=$ muy en desacuerdo) y para la última opción $(\mathrm{MA}=$ muy de acuerdo). Estadísticos descriptivos: $\mathrm{Mdn}=$ mediana, $\mathrm{RSI}=$ rango semiintercuartílico, $\mathrm{CAP}=$ coeficiente de asimetría percentílico de Kelly, $\mathrm{CPC}=$ coeficiente de curtosis percentílico corregido. Discriminabilidad: DM = diferencia media en el ítem entre los grupos de puntuaciones altas y bajas en la escala (suma de ítems puntuados en sentido de actitud favorable), ZU = valor del estadístico U de Mann-Whitney al comparar a los grupos de puntuaciones altas y bajas, significación de ZU en un contraste a dos colas: ns (no significativo) $p>.05, * p \leq .05, * * p \leq .01 * * * p \leq .001$. Consistencia interna: $r_{\mathrm{PS}}=$ correlación poliserial entre el ítem y el resto de la escala, $\lambda^{2}=$ comunalidad en la extracción de un factor por mínimos cuadrados no ponderados desde la matriz de correlación policórica, $\mathrm{y} \alpha$ ord $\mathrm{t}=$ coeficiente alfa ordinal de la escala tras eliminar el ítem. 
siendo la varianza compartida entre cada par de factores menor que la varianza media extraída de cada factor, por lo que los tres factores presentaron validez discriminante. La bondad de ajuste fue buena por siete índices, $\chi^{2} / g l=566.68 / 461=$ $1.23<2$; GFI $=.965$, $\mathrm{AGFI}=.960, \mathrm{CFI}=.991$, $\mathrm{NFI}=.954$ y $\mathrm{RFI}=.951>.95 ; \mathrm{y}$ RMSEA $=.024$ $<.05$, y aceptable por uno, SRMR $=.072<.075$.

Distribución de la puntación total de la EACIN-32 $y$ sus tres factores

Las distribuciones de la puntuación total $(\mathrm{DP}=4.21, p=.122)$ y CAII $(\mathrm{DP}=.18, p=.912)$ se ajustaron a una curva normal por la prueba de D'Agostino-Pearson. La distribución del factor ACID fue simétrica y unimodal, con una media $(\mathrm{M}=2.32)$ y mediana $(\mathrm{Mdn}=2.31)$ muy próxi- mas. Aunque presentó ligera leptocurtosis $(\mathrm{K}=$ .66, IC del 95\%: .18, 1.15), la hipótesis nula de distribución normal se pudo sostener por la prueba de D'Agostino-Pearson con un nivel de significación de .01. ( $\mathrm{DP}=8.30, p=.016)$. La distribución del factor COG presentó concentración en los valores altos ( $\mathrm{K}=1.29$, IC del 95\%: .80, 1.77) con colas asimétricas y sesgo hacia los valores por debajo de la mediana ( $\mathrm{Sk}=-.60$, IC del 95\%: $-.84,-.36)$, lo que no se ajusta a una curva normal $(\mathrm{DP}=50.81, p<.001)$.

\section{Comparación de medias entre los factores}

Debido al ajuste a la normalidad de la distribución en la puntuación total de la EACIN-32 y el factor CAII, una buena aproximación a la normalidad en el caso del factor ACID y

\section{Figura 1}

Modelo de tres factores correlacionados estimado por mínimos cuadrados no ponderados desde la matriz de correlación policórica.

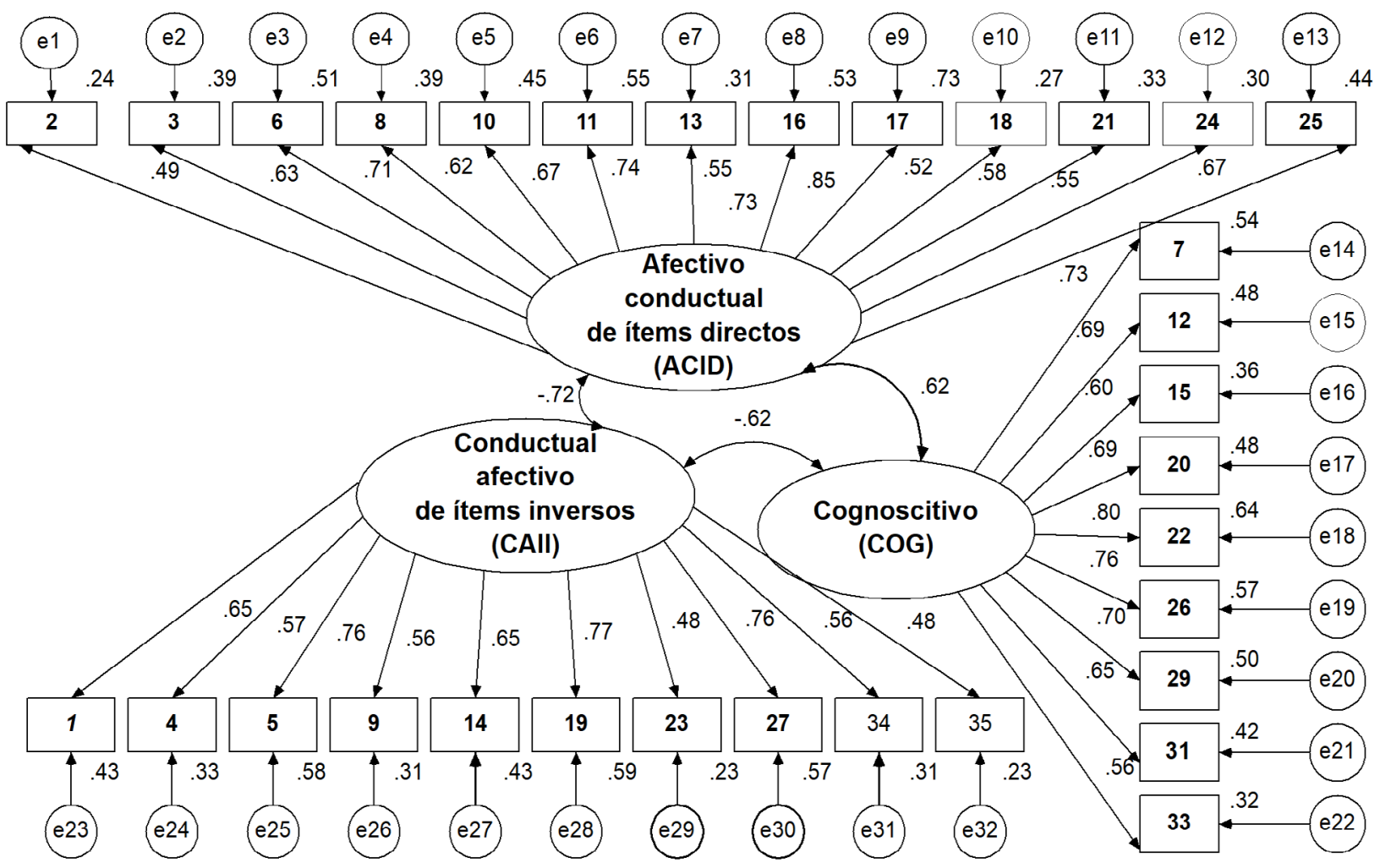


asimetría y curtosis leves en el factor COG, se hizo la comparación global de las medias a través del análisis de varianza de medidas repetidas. La hipótesis nula de esfericidad o independencia entre los residuos se mantuvo por la prueba de Mauschly: $\mathrm{W}=.99, \chi_{(2, \mathrm{~N}=392)}^{2}=2.26, p=.345$. Hubo diferencia significativa entre las medias de los tres factores $\quad\left(\mathrm{F}_{[2,324]}=546.71, p<.001\right)$ con una potencia unitaria y un tamaño del efecto muy grande, $\eta^{2}=.58$. Al hacer las comparaciones por pares a posteriori con la prueba $t$ de Student para muestras emparejadas con la corrección de Bonferroni sobre el nivel de significación $(2 * \alpha /$ $\left.\left[\mathrm{k}^{*}(\mathrm{k}-1)\right]=.1 / 6=.017\right)$, la media del factor cognoscitivo fue significativamente mayor que la del factor ACID $\left(t_{[391]}=32.65, p<.001\right)$ y la del factor CAII $\left(t_{[391]}=19.11, p<.001\right)$; en ambos casos con un tamaño del efecto grande ( $d$ de Cohen $=1.65$ y .97 , respectivamente). A su vez, la media del factor ACID fue significativamente mayor que la media del factor CAII $\left(t_{[391]}=13.68\right.$, $p<.001)$ con un tamaño del efecto moderado $(d$ de Cohen $=.69$ ).

Relación con las variables sociodemográficas y académicas

Al comparar las medias por la prueba $t$ de Student, en los cuatro casos, se pudo asumir la hipótesis de igualdad de varianzas por la prueba de Levene. La media de los 101 hombres ( $\mathrm{M}=$ 2.47, IC del 95\%: 2.35, 2.59) fue significativamente mayor que la media de las 291 mujeres (M $=2.27$, IC del 95\%: 2.20, 2.33) en el factor ACID $\left(t_{[390]}=3.07, p=.002\right)$. El tamaño del efecto del sexo sobre el factor ACID fue pequeño ( $g$ de Hedges $=.35$, IC del 95\%: .13, .58). Las medias entre hombres y mujeres fueron estadísticamente equivalentes en la puntuación total de EACIN-32 $\left(t_{[390]}=1.77, p=.078\right)$, el factor COG $\left(t_{[390]}=-.52\right.$, $p=.605)$ y el factor CAII $\left(t_{[390]}=.96, p=.339\right)$.

Las comparaciones de medias en la EACIN-32 y sus tres factores por el área profesional se hicieron por el análisis de varianza de una vía para grupos independientes. Sólo se compararon cinco grupos: área clínica, laboral, educativa, social y de investigación. Se excluyeron los casos de indecisos, o que habían señalado neuropsicología u otra opción por su baja frecuencia. Al mantenerse la hipótesis nula de igualdad de varianza entre los cinco grupos por la prueba de Levene $\left(\mathrm{F}_{[4,367]}\right.$ $=.25, p=.910$ para EACIN-32; $\mathrm{F}_{[4,367]}=.20, p=$ .939 para ACID; $\mathrm{F}_{[4,367]}=.21, p=.930$ para $\mathrm{COG}$, $\mathrm{F}_{[4,367]}=.28, p=.894$ para CAII), las comparaciones se realizaron con la prueba de Dunnett.

El área de trabajo a la que el estudiante piensa dedicarse tras la titulación tuvo un efecto significativo y de tamaño mediano sobre la puntuación total de la EACIN-32 $\left(\eta^{2}=.093\right)$ y sobre el factor ACID $\left(\eta^{2}=.102\right)$; fue de tamaño pequeño sobre el factor CAII $\left(\eta^{2}=.055\right)$ y el factor COG $\left(\eta^{2}=.036\right)$. Las cuatro comparaciones entre los grupos del área clínica, laboral, educativa y social con el grupo del área de investigación fueron significativas en la puntuación total $(p \leq .05)$ y el factor ACID. En el factor CAII, estas comparaciones fueron significativas $(p \leq .05)$ a excepción del grupo del área social y el de investigación ( $p$ $=.124)$. En el factor COG, sólo la comparación entre el grupo del área laboral y de investigación fue significativa $(p \leq .01)$. En todas las comparaciones, la media del grupo del área de investigación fue más alta. La media más baja fue la del área laboral tanto en la puntuación total como en los tres factores. El tamaño del efecto, al ser estimado por la $g$ de Hedges corregida de sesgo, fue grande en todas las comparaciones significativas ( $g$ de Hedges $=-0.94$ a -1.84) .

La puntuación total en la EACIN-32 y los factores ACID y CAII tuvieron correlaciones significativas, positivas y con una fuerza de aso- 
ciación débil con la calificación en la materia de investigación, la edad y el semestre cursado. El factor COG presentó correlación positiva y con una fuerza de asociación débil con la calificación promedio, pero fue independiente de las dos variables sociodemográficas. Las correlaciones de la edad con la EACIN-32 y los factores ACID y CAII no fueron significativas al parcializar la varianza del semestre cursado (Tabla 2).

\section{Discusión}

Se formuló como primer objetivo valorar si los 35 ítems de la EACIN presentaban problemas de efecto techo o suelo, falta de discriminabilidad y consistencia interna débil. Por los resultados del estudio original de Aldana et al. (2016) con estudiantes y maestros colombianos, se esperaba que los ítems 1, 6, 10, 18 y 31 mostraran una consistencia interna débil. En la presente muestra, ninguno de estos cinco ítems mostró problemas de consistencia interna, discriminabilidad o distribución con excesiva asimetría o curtosis; tampoco el nuevo ítem (ítem 35) sobre escaso tiempo dedicado a lectura presentó problemas. Aldana et al. (2016) con los 34 ítems obtuvieron una consistencia interna total alta por el coeficiente alfa de Cronbach. En el presente estudio la consistencia interna total fue mayor: con 35 o 34 ítems fue muy alta por el coeficiente alfa ordinal e incluso por el coeficiente de Cronbach.

Se estableció como segundo objetivo explorar la estructura factorial y verificar si los factores cumplían con criterios de consistencia interna, validez convergente y validez discriminante. Se esperaba una estructura de tres factores por los campos de contenido definidos a la hora de diseñar y seleccionar los ítems: afectivo, cognoscitivo y conductual (Aldana et al., 2016). Además, al formar parte de un mismo constructo, se esperaba que los tres factores estuvieran correlacionados y que cumplieran con criterios de consistencia interna, validez convergente y validez divergente. No se reprodujo la estructura esperada, pero sí una afín. El número de factores obtenidos fue tres. Los contenidos sobre aspectos afectivos y conductuales se unieron para diferenciarse por un aspecto formal de la dirección de la redacción de sus ítems: directa o inversa (ACID y CAII). El tercer factor se definió con los ítems sobre aspectos cognoscitivos (COG). Los tres factores

\section{Tabla 2}

Correlaciones con semestre cursado, edad y calificación en la materia de investigación.

\begin{tabular}{|c|c|c|c|c|}
\hline \multirow{2}{*}{ Escala y sus factores } & Semestre & Edad & Edad(sem) & Calificación \\
\hline & $r_{S}$ & $r_{s}$ & $r_{p}$ & $\boldsymbol{R}$ \\
\hline EACIN-32 & $\begin{array}{c}.110^{*} \\
(.011, .209)\end{array}$ & $\begin{array}{c}.129^{*} \\
(.030, .228)\end{array}$ & $\begin{array}{c}.075^{\mathrm{ns}} \\
(-.025, .174)\end{array}$ & $\begin{array}{c}.177^{* * *} \\
(.078, .270)\end{array}$ \\
\hline ACID & $\begin{array}{c}.103^{*} \\
(.004, .202)\end{array}$ & $\begin{array}{c}.130^{* *} \\
(.031, .229)\end{array}$ & $\begin{array}{c}.082^{\mathrm{ns}} \\
(-.017, .182)\end{array}$ & $\begin{array}{c}.156^{* *} \\
(.056, .253)\end{array}$ \\
\hline $\mathrm{COG}$ & $\begin{array}{c}.043^{\mathrm{ns}} \\
(-.056, .142)\end{array}$ & $\begin{array}{c}.051^{\mathrm{ns}} \\
(-.048, .150)\end{array}$ & $\begin{array}{c}.030^{\mathrm{ns}} \\
(-.070, .129)\end{array}$ & $\begin{array}{c}.109^{*} \\
(.010, .202)\end{array}$ \\
\hline CAII & $\begin{array}{c}.111^{*} \\
(.012, .210)\end{array}$ & $\begin{array}{c}.115^{*} \\
(.016, .214)\end{array}$ & $\begin{array}{c}.054^{\mathrm{ns}} \\
(-.045, .154)\end{array}$ & $\begin{array}{c}.169^{* * *} \\
(.067, .268)\end{array}$ \\
\hline
\end{tabular}

Nota. $r_{\mathrm{S}}=$ coeficiente de correlación por rangos de Spearman, $r_{\mathrm{p}}=$ coeficiente por rangos parcial, $r=$ coeficiente de correlación producto-momento de Pearson. Intervalo de confianza fijado en $95 \%$. Significación en un contraste a dos colas: ns (no significativa) $p>.05, * p \leq .01, * * p \leq .001, * * * p \leq .001$. 
correlacionaron, pero con buena validez discriminante. El factor ACID mostró una consistencia interna muy alta y los otros dos (COG y CAII) alta. Al especificarse cada factor con sus indicadores exactos, los tres factores claramente mostraron validez convergente y el ajuste del modelo fue bueno. Por tanto, la escala muestra validez de constructo estructural, y este modelo de tres factores se puede adoptar como hipótesis estructural para futuros análisis confirmatorios con muestras independientes. El presente estudio constituye una investigación exploratoria, ya que el análisis factorial confirmatorio se realizó en la misma muestra que el exploratorio (Byrne, 2016).

El tercer objetivo enunciado fue describir la distribución de la puntuación total de la EACIN-32 y sus factores. Muy vinculado a este se encuentra el cuarto objetivo sobre interpretar el nivel actitudinal promedio y comparar los promedios de los factores. La expectativa era que el nivel actitudinal fuese alto y muy alto con una distribución con sesgo hacia los valores altos, debido a la política y programas docentes de las facultades públicas que fomentan la investigación y una ciencia basada en evidencias (Aldana et al., 2016; Blanco \& Alvarado, 2005; De la Cruz, 2013; Pelcastre-Villafuerte, Gómez-Serrato, \& Zavala, 2015).

Se encontró ajuste a la distribución normal en las puntuaciones del factor CAII y en la puntuación total de la escala (EACIN-32) y una buena aproximación a la normalidad en el factor ACID con simetría y leve leptocurtosis. El factor COG mostró colas asimétricas, con su cola más larga hacia la izquierda, menor variabilidad relativa y una mayor concentración de lo esperado para una curva normal en los valores altos; no obstante, tanto la asimetría negativa como la leptocurtosis fueron leves y su perfil fue acampanado. Por tanto, esta escala de actitud y sus factores parecen presentar una distribución propia de rasgos adaptativos, en los que la mayoría (68\%) se concentra en torno a una desviación estándar de la media (expresión promedio o normal), una minoría se desvía por exceso $(16 \%)$ y otra minoría por defecto $(16 \%)$ de esta expresión media o con mayor éxito adaptativo (Lyon, 2012).

Si se divide el rango continuo de las puntuaciones en la escala y sus tres factores, de 0 a 4 , en cinco intervalos de amplitud constante ([valor máximo - valor mínimo]/número de ítems $=[4-$ $0] / 5=.8$ ) en correspondencia con los cinco valores ordinales de respuesta a los ítems, se puede interpretar el nivel actitudinal. Valores entre $0 \mathrm{y}$ .79 reflejan una actitud muy desfavorable hacia la investigación, entre .8 y 1.59 desfavorable, entre 1.6 y 2.39 neutra, entre 2.4 y 3.19 favorable y entre 3.2 y 4 muy favorable. Siguiendo este planteamiento de interpretación, la media en el factor COG $(\mathrm{M}=3.20)$ refleja una actitud promedio muy favorable, las medias en el total de escala y el factor ACID (M = 2.68 y 2.67, respectivamente) reflejan actitudes favorables y la media en el factor CAII $(\mathrm{M}=2.32)$ refleja una actitud neutra. Considerando que la media fue diferencial entre los tres factores, las aspectos cognitivos o intelectuales de la actitud hacia la investigación están más definidos que los aspectos prácticos o de conducta, lo cual es un fenómeno usual en valores transmitidos en ámbitos escolares y académicos, como ocurre con las conductas de salud sexual (Moral \& Ortega, 2009) y hábitos saludables (Brazil, Milagres, Duarte, \& Rocha, 2018).

El quinto objetivo consistió en evaluar la relación de la EACIN-32 con la edad y el sexo. Conforme con la expectativa, el promedio en la puntuación total y dos de los factores fue equivalente entre ambos sexos (Pelcastre-Villafuerte et al., 2015). Sólo hubo una diferencia significativa con un tamaño del efecto pequeño con un mayor 
promedio en el factor ACID en hombres que en mujeres. Precisamente, la lógica analítica e instrumental de la ciencia es más afín al rol de género masculino que al femenino. Probablemente, si se midiese y controlase el rol de género, la correlación resultaría espuria (Kumari \& Saraladevi, 2014). También, conforme con la expectativa se obtuvo una correlación positiva con la edad. Esta relación puede atribuirse a un mayor tiempo de exposición a los valores científicos inculcados por los maestros (Estrada-Corona, 2012; Vázquez \& Manassero, 2008), apoya esta interpretación el hecho de que la correlación dejó de ser significativa una vez se parcializó el semestre cursado. No obstante, al ser mayor la correlación con la edad que con el semestre, el hecho de tener una mayor madurez para la reflexión y adopción de dichos valores podría ser también un factor contribuyente.

El sexto objetivo fue aportar una prueba de validez de constructo al verificar si la escala presenta un mayor promedio entre los estudiantes que piensan dedicarse a la investigación y si tiene una correlación positiva con la calificación en la materia de investigación. Se cumplieron ambas hipótesis, lo que aporta evidencias de validez de constructo de tipo convergente a la escala. El tamaño del efecto del área de investigación sobre la puntuación total de la EACIN-32 fue mediano en la comparación total, por lo que resulta grande en las comparaciones por pares. La fuerza de asociación entre la puntuación de la EACIN-32 y la calificación promedio fue débil, por lo que probablemente otras variables como hábitos de estudio, promedio en las materias e inteligencia tengan asociación más fuerte.

Una primera limitación del estudio fue el uso de un muestreo no probabilístico. Por lo tanto, las inferencias con la debida precaución se limitan a la facultad de psicología en la que se recolectó la muestra. Aunque debe tenerse en cuenta que la tasa de casos fue grande, cubre más de un décimo de su población. Una segunda limitación fue el diseño ex post facto de corte transversal, que impide interpretar las asociaciones halladas en términos causales. Una tercera limitación fue que la actitud hacia la investigación se evaluó a través de un solo instrumento de autoinforme, por lo que las conclusiones se circunscriben a esta escala.

Se recomienda el uso de la EACIN-32 para la evaluación y estudio de la actitud hacia la investigación en contextos universitarios. Dado que la puntuación de la escala presenta un buen ajuste a la normalidad, se sugiere contrastar el modelo de tres factores correlacionados propuesto con una muestra probabilística y a su vez estimar los baremos de la escala, los cuales se pueden establecer a través de puntuaciones $T$. El muestreo se podría hacer con estudiantes universitarios. Se podrían aportar nuevas pruebas de validez de constructo concurrentes al verificar la correlación fuerte o muy fuerte de la EACIN-32 con otras escalas de actitud hacia la investigación, como las de Papanastasiou (2005) y Blanco y Alvarado (2005). De igual manera, se podría estudiar la relación de la EACIN-32 con la deseabilidad social. Se esperaría que el efecto de la deseabilidad social sea trivial o débil, y que esta tenga más efecto sobre el factor cognoscitivo, al ser más afín a los contenidos inculcados en las aulas y laboratorios por los maestros.

\section{Referencias}

Aldana, G. M., Caraballo, G. J., \& Babativa, D. A. (2016). Escala para medir Actitudes hacia la Investigación (EACIN): Validación de contenido y confiabilidad. Aletheia. Revista de Desarrollo Humano, Educativo y Social Contemporáneo, 8(2), 104-121. Recuperado de http://aletheia.cinde.org.co/index.php/ALETHEIA/article/view/325/253 
Aldana de Becerra, G. M., \& Joya-Ramírez, N. S. (2011). Actitudes hacia la investigación en docentes de metodología de la investigación. Tabula Rasa, 14, 295309. doi: $10.25058 / 20112742.428$

American Psychological Association (2017). Ethical principles of psychologists and code of conduct. With the 2016 amendment to standard 3.04. Washington, DC: American Psychological Association. Recuperado de https://www.apa.org/index

Ato, M., López, J. J., \& Benavente, A. (2013). Un sistema de clasificación de los diseños de investigación en psicología. Anales de Psicología, 29(3), 1038-1059. doi: 10.6018/analesps.29.3.178511

Blanco, N., \& Alvarado, M. E. (2005). Escala de actitud hacia el proceso de investigación científico social. Revista de Ciencias Sociales, 11(3), 537-544. Recuperado de http://produccioncientificaluz.org/index. $\mathrm{php} / \mathrm{rcs} / \mathrm{index}$

Borsboom, D., Mellenbergh, G. J., \& van Heerden, J. (2004). The concept of validity. Psychological Review, 111(4), 1061-1071. doi: 10.1037/0033295X.111.4.1061

Brazil, J. M., Milagres, M. P., Duarte, A. C. S., \& Rocha, R. M. (2018). Social representations of university students on healthy habits. Journal of Nursing UFPE on line, 12(1), 189-193. doi: 10.5205/1981-8963-v12i1a 23502p189-193-2018

Byrne, B. M. (2016). Structural equation modelling with amos. Basic concepts, applications, and programming ( $3^{\mathrm{a}}$. ed.). New York, NY: Routledge. doi: $10.4324 / 9781315757421$

Conover, W. J. (1999). Practical nonparametric statistics ( $3^{\mathrm{a}}$ ed.). New York, NY: John Wiley \& Sons.

De la Cruz, C. (2013). Actitudes hacia la investigación científica en estudiantes universitarios: Análisis en dos universidades nacionales de Lima. PsiqueMag, 2(1), 1-16. Recuperado de http://ojs.ucvlima.edu.pe/ index.php/psiquemag/article/view/12/12

Estrada-Corona, A. (2012). La actitud del individuo y su interacción con la sociedad. Entrevista con la Dra. María Teresa Esquivias Serrano. Revista Digital Uni- versitaria, 13(7), 1-12. Recuperado de http://www. revista.unam.mx

Gadermann, A. M., Guhn, M., \& Zumbo, B. D. (2012). Estimating ordinal reliability for Likert-type and ordinal item response data: A conceptual, empirical, and practical guide. Practical Assessment, Research \& Evaluation, 17(3), 1-13. Recuperado de http://pareonline.net

Gómez-Delgado, Y. A., Bazán-Ramírez, A., \& Villalobos-Galvis, F. H. (2017). Factores del estudiante que dificultan la enseñanza-aprendizaje de competencias de investigación. Interacciones. Revista de Avances En Psicología, 3(3), 101-110. doi: 10.24016/2017. v3n3.68

González-González, J., Galindo-Miranda, N. E., Galindo-Miranda, J. L., \& Gold-Morgan, M. (2004). Los paradigmas de la calidad educativa. De la autoevaluación a la acreditación. Ciudad de México: Unión de Universidades de América Latina.

Hesterberg, T. C., Moore, D. S., Monaghan, S., Clipson, A., \& Epstein, R. (2014). Bootstrap methods and permutation tests. En D. S. Moore, G. McCabe \& B. A. Craig (Eds.), Introduction to the practice of statistics ( $8^{\mathrm{a}}$. ed.). New York, NY: W.H. Freeman and Company.

IBM Corporation (2016). IBM SPSS Statistics for Windows, Version24.0 [software de cómputo]. Armonk, NY: IBM.

Kumari, S. N., \& Saraladevi, K. (2014). Gender differences in attitude towards science teaching and learning styles among adolescents. International Journal of Science and Research, 3(10), 50-58. Recuperado de https://www.ijsr.net

Lyon, A. (2012) Mathematical explanations of empirical facts, and mathematical realism. Australasian Journal of Philosophy, 90(3), 559-578, doi: 10.1080/00048402.2011.596216

Moral, J., \& Ortega, M. E. (2009). Representación social de la sexualidad y actitudes en estudiantes universitarios mexicanos. Revista de Psicología Social, 24(1), 65-79. doi: 10.1174/021347409786922998 
Papanastasiou, H. C. (2005). Factor structure of the "Attitudes Toward Research" Scale. Statistics Education Research Journal, 4(1), 16-26. Recuperado de https://iase-web.org/Publications.php?p=SERJ

Pelcastre-Villafuerte, L., Gómez-Serrato, A. R., \& Zavala, G. (2015). Actitudes hacia la ciencia de estudiantes de educación preuniversitaria del centro de México. Revista Eureka sobre Enseñanza y Divulgación de las Ciencias, 12(3), 475-490. doi: 10.25267/rev_eureka_ensen_divulg_cienc.2015.v12.i3.06

Poitras, G. (2006). More on the correct use of omnibus tests for normality. Economics Letters, 90(3), 304-309. doi: 10.1016/j.econlet.2005.08.016

Ruiz, J. (2010). Importancia de la investigación. Revista Cientifica, 20(2), 125-126. Recuperado de https:// www.redalyc.org/articulo.oa?id=95912322001

Téllez, A., García, C. H., \& Corral-Verdugo, V. (2015). Effect size, confidence intervals and statistical power in psychological research. Psychology in Russia: State of the Art, 8(3), 27-46. doi: 10.11621/ pir.2015.0303

Vázquez-Alonso, A., \& Manassero-Mas, M. A. (1997). Una evaluación de las actitudes relacionadas con la ciencia. Enseñanza de las Ciencias, 15(2), 199-213. Recuperado de http://www.raco.cat/index.php/Ensenanza

Vázquez, A., \& Manassero, M. A. (2008). El declive de las actitudes hacia la ciencia de los estudiantes: Un indicador inquietante para la educación científica. Revista Eureka sobre Enseñanza y Divulgación de las Ciencias, 5(3), 274-292. doi: 10.25267/Rev_Eureka_ensen_divulg_cienc.2008.v5.i3.03 


\section{Anexo. EACIN-32}

A continuación encontrarás una serie de afirmaciones relacionadas con la investigación (clases de investigación, análisis de datos, etc.), por favor marque con una $\mathrm{X}$ la respuesta con la cual te sientas más identificado(a). No medites mucho tu respuesta, no hay respuestas buenas ni malas. Las opciones de respuesta son:

$\mathrm{O}=$ Muy en desacuerdo, $1=$ En desacuerdo, 2 = Ni de acuerdo ni en desacuerdo,

$3=$ De acuerdo, $4=$ Muy de acuerdo

\begin{tabular}{|c|c|c|c|c|c|}
\hline & $\mathbf{0}$ & 1 & 2 & 3 & 4 \\
\hline $\begin{array}{l}\text { 1. En mi opinión en la universidad no deberían enseñar investigación. } \\
\text { 2. En los eventos de investigación (congresos, encuentros) me relaciono con la } \\
\text { gente. } \\
\text { 3. De las cosas que más me agradan son las conversaciones científicas. } \\
\text { 4. Eso de estar tomando cursos de actualización no es para mí. } \\
\text { 5. Creo que estar consultando información científica es perder el tiempo. } \\
\text { 6. Considero que tengo la paciencia necesaria para investigar. } \\
\text { 7. Todos los profesionales deberían aprender a investigar. } \\
\text { 8. La mayoría de las cosas me generan curiosidad. } \\
\text { 9. Casi siempre dejo para después lo que tiene que ver con investigación. } \\
\text { 10. Busco informarme de los temas de actualidad. } \\
\text { 11. Me gusta capacitarme para adquirir habilidades de investigación. } \\
\text { 12. Creo que la persistencia contribuye a alcanzar las metas. } \\
\text { 13. Acostumbro a escribir para profundizar en temas de interés. } \\
\text { 14. Las actividades de investigación diarias no me inspiran nada novedoso. } \\
\text { 15. Investigar es posible si tenemos interés de hacerlo. } \\
\text { 16. Con frecuencia me encuentro consultando información en artículos científicos. } \\
\text { 17. La investigación es una de las cosas que me despierta interés. } \\
\text { 18. Soy ordenado(a) en mis actividades de investigación. } \\
\text { 19. Las conversaciones científicas me parecen aburridas. } \\
\text { 20. Trabajar con otros en investigación nos ayuda a alcanzar mejores resultados. } \\
\text { 21. Se me ocurren ideas innovadoras acerca de problemas cotidianos. } \\
\text { 22. Considero que la investigación ayuda a detectar errores de la ciencia. } \\
\text { 23. Para ser sincero(a) realmente lo que menos hago es escribir. } \\
\text { 24. Aprovecho cualquier oportunidad para dar a conocer mis trabajos de la escuela } \\
\text { relacionados a investigación. } \\
\text { 25. Me gusta agilizar los trabajos relacionados con investigación. } \\
\text { 26. Para mí, en investigación es importante fortalecer la capacidad de escuchar. } \\
\text { 27. Pensar en ponerme a investigar me produce desánimo. } \\
\text { 29. En mi opinión, sin investigación la ciencia no avanzaría. } \\
\text { 31. A mi parecer la investigación contribuye a resolver problemas sociales. } \\
\text { 33. Reconozco que la investigación ayuda a corregir errores del sentido común. } \\
\text { 34. Soy el último en enterarse de los temas de actualidad. } \\
\text { 35. Para ser sincero(a) realmente lo que menos hago es leer. } \\
\text { a }\end{array}$ & & & & & \\
\hline
\end{tabular}

